\title{
Immune checkpoint inhibitors and rheumatic adverse events
}

\author{
Ioana Cretu ${ }^{1,2}$, Mihai Bojinca ${ }^{1,2}$, Mihaela Milicescu ${ }^{1,2}$, Bogdan Cretu ${ }^{1,4}$, \\ Ruxandra Ionescu ${ }^{1,3}$ \\ 1"Carol Davila" University of Medicine and Pharmacy, Bucharest, Romania \\ 2Department of Internal Medicine and Rheumatology, \\ "Dr. Ion Cantacuzino" Hospital, Bucharest, Romania \\ ${ }^{3}$ Department of Internal Medicine and Rheumatology, \\ "Sf. Maria" Clinical Hospital, Bucharest, Romania \\ ${ }^{4}$ Department of Orthopaedics and Traumatology, Emergency University Hospital \\ of Bucharest, Romania
}

\begin{abstract}
Immune checkpoint inhibitors are used with increasing regularity in metastatic disease. Anti-CTLA4 (ipilimumab) anti-PD1 (pembrolizumab, nivolumab) or anti-PD-L1 (avelumab, atezolizumab) are approved for use in metastatic melanoma, non-small cell lung cancer, renal cell carcinoma and other malignancies. Despite their advantages, their immune adverse reactions are important. This paper is a review current literature regarding rheumatologic adverse reactions that follow $\mathrm{ICl}$ treatment. Their frequency is not high but it is important to correctly diagnose and treat them. Most of the adverse reactions are mild and moderate. The treatment for immune related adverse events (IRAEs) is currently not supported by a sufficient amount of studies. The best treatment option is therefore based on the rheumatologist experience.
\end{abstract}

Keywords: immune checkpoint inhibitors, immune related adverse events, rheumatic disease

\section{INTRODUCTION}

Immunotherapy treatment helps the immune system fight against different diseases and is used in the treatment of different types of cancer. For example, the immune checkpoint blockade has been used in the treatment of cancer. It enhances antitumor immunity by blocking intrinsic down regulators of immunity, such as cytotoxic T-lymphocyte antigen 4 (CTLA-4), programmed cell death 1 (PD-1) or its ligand, programmed cell death ligand 1, (PD-L1). Some of the antibodies that provide the immune blockade (Immune checkpoint inhibitors - ICI's) have proven their efficiency in cancer treatment and are approved by FDA. Ipilimumab (CTLA-4) and Pembrolizumab (PD-1) are used in the treatment of advanced melanoma such as metastatic disease and also in other tumor types including non-small-cell lung cancer (NSCLC) and advanced renal carcinoma (1).

ICI's produce effective antitumor immune response, but they also modify the immunologic ho- meostasis by blocking the negative regulators of immunity. This can be associated with inflammatory adverse effects known as immune related adverse events (IRAEs). These adverse effects can arise in any organ, but most frequently in the following organs are: gastrointestinal tract, the liver, endocrine system and skin. Neurologic, cardiovascular, pulmonary, musculoskeletal and hematologic adverse effects are less frequent. In some cases, IRAE's will lead to temporary or permanent ending of cancer treatment, corticosteroid therapy or immunosuppressive therapy. Taking into account the vast majority of systems that can be affected by IRAEs, the multidisciplinary approach is the key in the diagnosis, treatment and follow-up of them $(2,3)$.

The purpose of this review is to evaluate the actual knowledge regarding IRAEs after ICI's in the terms of diagnosis and treatment. We will discuss the rheumatologic diseases IRAEs after ICI's, they are less frequent than other adverse effects, but their morbidity is significant. 


\section{PATHOPHYSIOLOGY OF IMMUNE-RELATED ADVERSE EVENTS}

The actual knowledge regarding pathophysiology underlying IRAEs is not completely understood but it is attributed to the disruption of the immunologic homeostasis (2). CTLA-4 plays an important role in inhibiting the immune response by attenuating T-cell activation at the beginning in the immune response (4). PD-1 inhibits T cells in a late faze of the immune response in the peripheral tissues. From the different functions of CTLA- 4 and PD- 1 we can explain why the adverse reactions are different. Studies by Tivol EA et al. and Waterhouse P et al. found that in mice without CTLA-4 gene die from lymphoproliferation (5), while a study by Nishimura $\mathrm{H}$ et al. found that mice without PD-1 gene suffered from immune adverse reactions like arthritis and cardiomyopathy (6).

The patients treated with anti-CTLA-4 and with anti-CD1 have different adverse reactions, the effects of anti-CTLA-4 therapy being more severe. In the patients with anti-CTLA-4 it was found that hypophyzitis is more common. It is not totally known why organ specific adverse reactions occur, but it has been recently discovered that the hypophysis has expression of CTLA-4 on normal pituitary cells (1). On the other hand, in patients with anti-PD 1 pneumonitis and thyroiditis appear to be more common. PD-1 has another major importance in maintaining self-tolerance: it doesn't allow the immune system to attack its own organism $(7,8)$.

The frequent use of ICI's should increase the rheumatologist's awareness regarding the immune adverse reactions (9). The incidence of IRAEs depends on the type of the treatment and the type of malignancy $(10,11)$. The most frequent adverse reactions are the rash that is encountered in $30-35 \%$ of the patients treated for melanoma, dermatologic adverse reactions (vitiligo, neutrophilic dermatoses, pruritus and toxic epidermal necrolysis) (12).

In the pathophysiology of IRAEs, cytokines could also be involved. A study by Callahan M, et al showed that induced colitis in patients with ipilimumab is correlated with high levels of interleukin-17. This information can help treating the induced colitis with interleukin-17 blockade therapy like secukinumab, ixekizumab, and brodalumab $(13,14)$.

Preclinical in vivo studies on mice showed that the administration of anti-PD-1 and anti-PD-L1 de- termines tumoral growth inhibition and inhibition of metastatic disease. If anti-CTLA-4 and anti-PD-1 are used together, the antitumor effect is higher when compared to usage of either individually $(15,16,17)$.

\section{MUSCULOSKELETAL AND RHEUMATIC IRAES}

The main difference between conventional chemotherapy and immune checkpoint inhibitors is that when the immune system is stimulated, immune related adverse reactions can occur which will lead to the need of treatment turn off or administration of immune suppressor therapy. Despite the possibility of IRAEs occurrence, ICI's produce effective antitumor immune response in specific situations $(18,19)$.

IRAEs are frequent; they occur in $90 \%$ of patients when anti-CTLA- 4 are used and in $70 \%$ of patients that will use anti-PD-1 therapy will suffer from adverse reactions (20). The most common articular IRAEs is joint pain with or without inflammatory arthritis. Articular manifestations can occur at any time during ICI's (21). In most cases they will appear after 3 to 6 months of treatment with ICI's. It is important to mention that the toxicity of anti-CTLA-4 is dose-dependent while the toxicity of anti-PD-1/PDL-1 is not dose dependent (22).

\section{a. Arthralgia and inflammatory arthritis}

In the patients with ICI's treatment arthralgia and inflammatory arthritis are common adverse reactions. There are no prospective studies that followed the patients with inflammatory arthritis confirmed by a rheumatologist (18). An observational study done before and after the treatment that followed patients treated with anti-CTLA-4 with CT/PET-CT it was found out that in 4 patients from 119 the arthritis occurred (23).

The incidence of arthralgia is between 9 and $12 \%$, with a frequency of between 6 to $8 \%$ in patients that receive pembrolizumaz and nivolumab, $5 \%$ with ipilimumab and $10 \%$ with combined therapy (24).

Inflammatory arthritis can appear in small and large joints. Clinic manifestations can arise as monoarthritis, oligoarthritis and polyarthritis $(25,26)$. Small joint manifestations are similar to those from rheumatoid arthritis. Oligoarthritis manifestations are similar to reactive arthritis. There is a case report that correlated the appearance of psoriasis arthritis with the administration of nivolumab (21). 
The diagnosis is based on clinical facts, mainly on patient history. If there are no criteria for an inflammatory articular disease the diagnosis is of ICI's induced arthritis. Blood work can identify the inflammatory syndrome (CRP, high ESR), and in some patients the rheumatoid factor is positive (21).

\section{b. Myalgia and inflammatory myositis}

The second most common musculoskeletal adverse reaction after ICI's administration are Myalgia and inflammatory myositis. After administration of anti-PD-1 there are described cases of inflammatory myositis (23).

The majority of IRAEs occur after several months of treatment but after single administration of Nivolumab there have been described cases of myositis and myocarditis (27).

In a patient with metastatic melanoma treated with Ipilimumab, dermatomyositis occurred (28).

There there have been described cases of eosinophilic fasciitis which can mimic the symptoms of myositis (28).

\section{c. Systemic lupus erythematosus}

Systemic lupus erythematosus it is not usually associated with ICI's treatment. There has been a single description of a case of lupus nephritis after administration of Ipilimumab (23).

Lupus-like adverse reactions have been described in a case of Pembrolizumab administration for metastatic melanoma and in a case of metastatic pulmonary cancer after administration of Nivolumab $(29,30)$.

\section{d. Sicca syndrome}

Dryness of the eye and mouth occur in 3-24\% of patients treated with ICI's. A study that followed patients treated with Nivolumab, Ipilumumab and combined therapy found 4 cases of severe Sjögren syndrome $(9,31)$. Adverse reactions related to the mouth were severe in all 4 patients. There were no positive antibodies anti-Ro, and a patient had positive anti-La/SS-B antibodies and hypo echogenic foci in the parathyroid glands (32).

\section{e. Vasculitis}

There have been isolated cases of vasculitis after ICI's treatment. It has appeared in 3 cases after Ipilimumab administration and in 2 cases after Pembrolizumab administration (30). All the cases with vasculitis had associated skin adverse reactions and neurologic symptoms (9).
Cases of peripheral neuropathy have been described, with histopathologic exams. Such case occurred in patient with rheumatoid polyarthritis with a metastatic melanoma that was treated with Pembrolizumab (23).

\section{f. Polymyalgia rheumatica (PMR) - 6}

A study conducted at Cleveland Clinic which monitored patients treated with ICI's found in a case series that 3 out of 15 patients complained of PMR specific symptoms like pain and stiffness of the shoulder, hips and in the cervical region. None of the patients complained of specific symptoms of giant cell arteritis (23).

\section{TREATMENT}

The current treatment for IRAEs is not supported by enough studies. The best treatment option is based on the rheumatologist experience $(33,34)$.

Most of the IRAEs are treated with corticoids or other immunosupressors, for example Infliximab. Immunosuppressive treatment does not interfere with the efficacy of the ICIS and after a correct management most of IRAEs resolve (35).

For patients who receive corticosteroids for a long period of the time, $(20 \mathrm{mg}$ of prednisone for more than 4 weeks) prophylaxis for Pneumocystis Carinii must be taken into account (treatment of the immune related adverse events) (18).

The toxicity is less after administration of anti-PD-1 and anti-PDL-1 than after anti-CTLA-4 (18). The severity of the adverse reactions can be graded by the Common Terminology Criteria for Adverse Events (CTCAE) on a scale from 1 to 5 (1=mild, $2=$ moderate, $3=$ severe, $4=$ life threatening, $5=$ death related to toxicity), grading done by National Cancer Institute in 2009. ICI's are interrupted in case of grade 2 and are restarted when the symptoms/biological data indicate grade 1 or less (23).

Corticosteroids must be initiated if the symptoms persist more than 1 week.

For the grade 3 and 4, corticosteroids are administered in high doses and then reduced as the symptoms decrease.

If the symptoms persist after 3 days of intravenous corticosteroids administration, other immunosuppressive treatments like Infliximab must be taken into account.

For grade 4 toxicity ICI's treatment must be stopped permanently (Current Diagnosis and Management of Immune Related Adverse Effects) (18). 
There are concerns regarding the long-term effects of the immunosuppressive medication upon malignancy progression. Infliximab has been used for short periods of time for induced colitis after ICI's in patients with metastatic melanoma (36).

There is no indication for the use of Abatacept, because its opposite effect compared to ICI's (37). Because of the colitis risk after ICI's administration, there are concerns regarding Tocilizumab and Tofacitinib (IL-6 and JAK-inhibitor can determine the perforation of the colon) (38).

Non-biologic disease-modifying antirheumatic drugs like Sulfasalazine, Methotrexate, Hydroxiclochin can be taken into account for treatment because of the low tumoral risk (Immune-Related Adverse Effect of Cancer Immunotherapy) (9).

After the diagnosis of IRAEs it is recommended to start corticosteroids therapy with Methotrexate administration if the symptoms do not permit lowering the corticosteroid doses. Rheumatologic IRAEs have the tendency to become chronic so that is why is indicated to use the lowest dose of corticosteroids and the safest DMARD (39).

For patients with active malignancies, the use of anti-TNF must be careful because of the infection risk associated with biological therapy $(40,41)$. The use of biological agents has to be the second line of treatment if the Methotrexate fails (42).

\section{DISCUSSION}

In the past years, oncologists have encountered numerous IRAEs, differing from the adverse reactions given by conventional chemotherapy. Rheumatologic IRAEs have been poorly studied, a fact that has led to no consensus upon diagnostic and treatment by oncologists and rheumatologists.

The true incidence of IRAEs is hard to estimate, as it is known that $10 \%$ of patients treated with ICI's will develop rheumatologic adverse reactions. Most of the adverse reactions are mild and moderate, with the exception of severe myositis (23).

The detection of the antibodies in the management of IRAEs is poorly defined. For precise diagnosis and follow-up specific antibodies should be detected $(9,43)$.

Depending on the severity of the adverse reactions different treatments are applied. Inflammatory arthritis can be treated with NSAIDS or corticosteroids, while in other cases DMARD's and biologic treatment are better options $(44,45)$.

There are no prospective studies regarding the appropriate treatment of IRAEs, so the clinical experience of each rheumatologist and oncologist will dictate the optimal treatment. The attenuation of the severity of IRAEs is related to a good clinical evaluation and a proper management. Prolonged immunosuppression is associated with the risk of different opportunistic infections requiring antibiotic treatment (43).

Reducing the anti-PD-1 or ipilimumab doses has no clinical proven efficiency therefore it is not recommended for treating the toxicity (2).

Better management and diagnosis of IRAEs will provide a safer setting for ICI's treatment.

ICI's treatment provides good long-term results in patients with metastatic disease, the follow-up in those patients for IRAEs has to be on long term because the adverse reaction can occur years after the start of ICI's treatment. There is no clinical data about the safety of long term use of anti-CTLA4 and anti-PD1 which means that the patients will have to be monitored long term.

Clinical studies for evaluating ICI's have excluded the patients with preexisting autoimmune conditions, meaning that the evolution of these patients it is also unknown (46).

\section{CONCLUSIONS}

Immunotherapy is increasingly used in cancer treatment, and it is considered in some studies safer than chemotherapy. The downside of its use are the immune adverse reactions than can occur with a high incidence, warranting proper management. Most of the adverse reactions are reversible, unlike the endocrine adverse reactions which are permanent. IRAEs rarely are the cause of death in those patients. After those adverse reactions occur, it is important to recognize them and manage them properly, so the the patients' quality of life can be improved.

The current knowledge of the physiopathology of immune adverse reactions and how to diagnose and treat them must be improved. Strategies are needed to identify the patients at risk to develop adverse reactions. 


\section{REFERENCES}

1. Postow MA, Callahan MK, Wolchok JD. Immune Checkpoint Blockade in Cancer Therapy. Jurnal of Clinical Oncology. 2015;33:1974-1982

2. Postow MA, Sidlow R, Hellman MD. Immune-Related Adverse Events Associeted with Immune Checkpoint Blockade. The New England J of Med. 2018;378:158-68

3. Weber JS, Hodi FS, Wolchok JD et al. Safty profile of nivolumab monotherapy: A pooled analysis of patients with advanced melanoma. J Clin Oncol. 2017;35:785-92

4. Krummel MF, Allison JP. CTLA-4 engagement inhibits IL-2 accumulation and cell cycle progression upon activation of resting $T$ cells. J Exp Med 1996; 183:2533-40

5. Boussiotis VA. Molecular and biochemical aspects of the PD-1 checkpoint pathways. N Engl J Med. 2016;375:1767-78

6. Waterhouse P, Penninger JM, Timms E et al. Lymphoproliferative disorders with early lethality in mice deficient in CTLA-4. Science.1995;270:985-8

7. Blansfiield JA, Beck KE, Tran K et al. Cytotoxic T-lymphocyteassociated antigen-4 blockage can induce autoimmune hypophysitis in patients with metastatic melanoma and renal cancer. J Immunother. 2005;28:593-598

8. Dillard T, Yedinak CG, Alumkal J et al. Anti CTLA-4 antibody therapy associated autoimmune hypophysitis. Serious immune related adverse events across a spectrum of cancer subtypes. Pituitary. 2010; 13:29-38

9. Cappelli LC, Shah AA, Bingham CO. Immune-Related Adverse Effects of Cancer Immunotherapy - Implications for Rheumatology. Rheum Dis Clin N Am. 2017;43:65-78

10. Le DT, Uram JN, Wang H et al. PD-1 Blockade in tumors with mismatch-repair deficiency. N Engl J Med.2015;372(26):2509-20

11. Weber JS, Yang JC, Atkins MB et al. Toxicities of immunotherapy for the practioner. J Clin Oncol. 2015;33(18):2092-9

12. Brahmer J, Reckcamp KL, Baas $P$ et al. Nivolumab versus docetaxel in advanced squamous-cell non-small-cell lung cancer. $N$ Engl J Med. 2015;373(2):123-35

13. Motzer RJ, Escuidier B, McDermott DF et al. Nivolumab versus everolimus in advanced renal cell carcinoma. N Engl J Med.2015;373(19):1803-13

14. Robert C, Schachter J, Long GV et al. Pembrolizumab versus ipilimumab in advanced melanoma. $N$ Engl $\mathrm{J}$ Med. 2015;372(26):2521-32

15. Medina PJ, Adams VR. PD-1 Pathway Inhibitors: ImmunoOncology Agents for Restoring Antitum or Immune Responses. Pharmacotherapy. 2016;36 (3):317-334

16. Pardoll DM. The Blockade of immune checkpoints in cancer immunotherapy. Nat Rev Cancer. 2012;12:252-64.

17. Sharma P, Allison JP. Immune checkpoint targeting in cancer therapy: toward combination strategies with curative potential. Cell. 2015;161:205-14

18. Kumar V, Chaudhary N, Garg M et al.Current Diagnosis and Management of Immune Related Adverse Events (irAEs) Induced by Immune Checkpoint Inhibitor Therapy. Front Pharmacol. 2017;8 (49):1-13

19. Cappelli L, Gutierrez AK, Baer AN et al. Inflamatory arthritis and sicca syndrome induced by nivolumab and ipilimumab. Ann Rheum Dis. 2016;76:43-50

20. Gangadhar TC, Vonderheide RH. Mitigating the toxic effects of anticancer immunotherapy. Nat Rev Oncol. 2014;11:91-99

21. Cappelli L, Bingham CO, Shah AA. Rheumatologic complications of checkpoint inhibitors immunotherapy. Up to date. 2018

22. Horvat TZ, Adel NG, Dang TO et al. Immune-related adverse events, need for systemic immunosuppression and effects on survival and time to treatment failure in patients with melanoma treated with ipilimumab at memorial sloan kettering cancer center. J Clin Oncol. 2015;33:3193

23. Benfaremo D, Mnfredi L, Luchetti MM et al. Musculoskeletal and Rheumatic Disease Induced by Immune Checkpoint Inhibitors: A Review of the Literature. 2018;13 (3):1-15

24. Spain L, Diem S, Larkin J. Management of toxicities of immune checkpoint inhibitors. Cancer Treat Rev. 2016;44:51-60

25. Cappelli LC, Gutirez AK, Baer AN et al. Inflammatory arthritis and sicca syndrome induced by nivolumab and ipilimumab.Ann Rheum Dis. 2017;76(1):43-50
26. Calabrese C, Kirchner E, Kontzias K et al. Rheumatic immunerelated adverse events of checkpoint therapy for cancer. Case series of a new nosological entity. RMD Open.2017;3(1)

27. Michot JM, Bigenwald C, Champiat S et al. Immunerelated adverse events with immune checkpoint blockade: a comprehensive review. European J of Cancer. 2016;54:139-148

28. Hodi FS, O Day SJ, McDermott DF et al. Improved survival with ipilimumab in patients with metastatic melanoma. $N$ Engl $\mathrm{J}$ Med. 2010;363:711-23

29. Fadel F, Karoui KE, Knebelmann B et al. Antibody Induced Lupus Nephritis. N Engl J Med. 2009;361(2):211-2

30. Perez-De-Lis M, Retamozo S, Flores-Chavez A et al. Autoimmune diseases induced by biological agents. A review of 12,731 cases (BIOGEAS Registry). Expert Opin Drug Saf. 2017;16(11):1255-71

31. Abdel-Rahman $\mathrm{O}$, Oweira $\mathrm{H}$, Petrausch $\mathrm{U}$ et al. Immune-related ocular toxicities in solid tumor patients treated with immune checkpoint inhibitors: a systematic rewiew. Expert Rev Anticancer Ther. 2017;17(4):387-94

32. Le Burel S, Champiat S, Mateus $C$ et al. Prevalence of immunerelated systemic adverse events in patients treated with anti-Programmed cell Death-Ligand 1 agents: A single center pharmacovigilance database analysis. Eur J Cancer. 2017;82:34-44

33. Friedman CF, Proverbs-Singh TA, Postow MA. Treatment of the Immune-Related Adverse Effects of Immune Checkpoint Inhibitors. JAMA Oncol. 2016;2(10):1346-53

34. Postow MA, Chesney J, Pavlicl AC et al. Nivolumab and ipilimumab versus ipilimumab in untreated melanoma. $N$ Engl $\mathrm{J} \mathrm{Med.}$ 2015;372(21):2006-2017

35. Weber JS, Antonia SJ, Topalian SL et al. Safety profile of nivolumab (NIVO) in patients (pts) with advanced melanoma (MEL): a pooled analysis. J Clin Oncol. 2015;33(15 suppl):abstr 9018

36. Turesson C, Matteson EL. Malignancy as a comorbidity in rheumatic disease. Rheumatology (Oxford).2013;52(1):5-14

37. Stone JH, Holbrook JT, Marriott MA et al. Solid malignancies among patients in the Wegener s Granulomatosis Etanercept Trial. Arthritis Rheum. 2006;54(5):1608-18

38. Johnston RL, Lutzky J, Chodhry A et al.Cytotoxic T-lymphocyteassociated antigen 4 antibody-induced colitis and management with infliximab. Dig Dis Sci. 2009;54(11):2538-40

39. Lidar M, Giat E, Garelick D et al.Rheumatic manifestation among cancer patients treated with immune checkpoint inhibitors. Autoimmunity Reviews (Elsevier).2018;17:284-289

40. Mercer LK, Askling J, Raaschou P, Dixon WG et al. Risk of invasive melanoma in patients with rheumatoid arthritis treated with biologics: Results from a collaborative project of 11 European biologic registers. Ann Rheum Dis. 2017;76:386-91

41. Giat E, Ehrenfeld M, Shoenfeld Y. Cancer and autoimmune disease. Autoimmune Rev. 2017;16:1049-57.

42. Buchbinder R, Barber $M$, Heuzenroeder $L$ et al.Incidence of melanoma and other malignancies among rheumatoid arthritis patients treated with methotrexate. Arthritis Rheum. 2008;59:794-9

43. Kroschinsky F, Stolzel F, Simione von Bonin et al. New drugs, new toxicities: severe side effects of modern targeted and immunotherapy of cancer and their management. Critical Care. 2017;21:89

44. Kostine M, Rouxel L, Barnetche T et al. Rheumatic disorders associated with immune checkpoint inhibitors in patients with cancer - clinical aspects and relationship with tumor response: A single-center prospective cohort study. Ann Rheum Dis. 2018;77(3):393-398

45. Kim ST, Tayar J, Trinh VA et al. Successful treatment of arthritis induced by checkpoint inhibitors with tocilizumab: A case series. Ann Rheum Dis. 2017;76(12):2061-2064

46. Menzies AM, Johnson DB, Ramanujam S et al. Anti-PD-1 therapy in patients with advanced melanoma and preexisting autoimmune disorders of major toxicity with ipilimumab. Ann Oncol. 2017;28(2):368-76 